\title{
Hom-left-symmetric color dialgebras, Hom-tridendriform color algebras and Yau's twisting generalizations
}

\author{
Ibrahima Bakayoko ${ }^{1} \cdot$ Sergei Silvestrov ${ }^{2}$ (D)
}

Received: 9 February 2020 / Accepted: 2 January 2021 / Published online: 20 February 2021

(c) The Author(s) 2021

\begin{abstract}
The goal of this paper is to introduce and give some constructions and study properties of Hom-left-symmetric color dialgebras and Hom-tridendriform color algebras. Next, we study their connection with Hom-associative color algebras, Hom-post-Lie color algebras and Hom-Poisson color dialgebras. Finally, we generalize Yau's twisting to a class of color Hom-algebras and use endomorphisms or elements of centroids to produce other color Homalgebras from given one.
\end{abstract}

Keywords Hom-tridendriform color algebra $\cdot$ Hom-associative color algebra · Hom-left-symmetric color dialgebra $\cdot$ Hom-Poisson color dialgebra

Mathematics Subject Classification 17D30 $\cdot$ 17B61 $\cdot$ 17A30 $\cdot 17$ A32

\section{Introduction}

The investigations of various $q$-deformations (quantum deformations) of Lie algebras began a period of rapid expansion in 1980's stimulated by introduction of quantum groups motivated by applications to the quantum Yang-Baxter equation, quantum inverse scattering methods and constructions of the quantum deformations of universal enveloping algebras of semisimple Lie algebras. In [2,19-25,31,32,39-41] various versions of $q$-deformed Lie algebras appeared in physical contexts such as string theory, vertex models in conformal field theory, quantum mechanics and quantum field theory in the context of $q$-deformations of infinitedimensional algebras, primarily the $q$-deformed Heisenberg algebras, $q$-deformed oscillator algebras and $q$-deformed Witt and $q$-deformed Virasoro algebras, and some interesting $q$ -

\footnotetext{
Sergei Silvestrov

sergei.silvestrov@mdh.se

Ibrahima Bakayoko

ibrahimabakayoko27@gmail.com

1 Département de Mathématiques, Université de N'Zérékoré, BP 50, N'Zérékoré, Guinea
}

2 Division of Mathematics and Physics, School of Education, Culture and Communication, Mälardalen University, Box 883, 72123 Västerås, Sweden 
deformations of the Jacobi identity for Lie algebras in these $q$-deformed algebras were observed.

Hom-Lie algebras and more general quasi-Hom-Lie algebras were introduced first by Larsson, Hartwig and Silvestrov [30], where the general quasi-deformations and discretizations of Lie algebras of vector fields using more general $\sigma$-derivations (twisted derivations) and a general method for construction of deformations of Witt and Virasoro type algebras based on twisted derivations have been developed, initially motivated by the $q$-deformed Jacobi identities observed for the $q$-deformed algebras in physics, along with $q$-deformed versions of homological algebra and discrete modifications of differential calculi. The general abstract quasi-Lie algebras and the subclasses of quasi-Hom-Lie algebras and Hom-Lie algebras as well as their color algebras generalizations have been introduced [30,35-38,56]. Subsequently, various classes of Hom-Lie admissible algebras have been considered in [49]. In particular, in [49], the Hom-associative algebras have been introduced and shown to be Hom-Lie admissible, that is leading to Hom-Lie algebras using commutator map as new product, and in this sense constituting a natural generalization of associative algebras, as Lie admissible algebras leading to Lie algebras via commutator map as new product. In [49], moreover several other interesting classes of Hom-Lie admissible algebras generalising some classes of non-associative algebras, as well as examples of finite-dimensional Hom-Lie algebras have been described. Since these pioneering works $[30,35-38,49]$, Hom-algebra structures have developed in a popular broad area with increasing number of publications in various directions. In Hom-algebra structures, defining algebra identities are twisted by linear maps. Hom-algebras structures are very useful since Hom-algebra structures of a given type include their classical counterparts and open more possibilities for deformations, extensions of homology and cohomology structures and representations, Hom-coalgebra, Hom-bialgebras and Hom-Hopf algebras (see for example $[1,3-11,18,26,27,29,33-35,50,53-55,58,60,63]$ and references therein).

Dendriform algebras were introduced by Loday as algebras with two operations which dichotomize the notion of associative algebras [42]. They are connected to K-theory, Hopf algebras, homotopy Gerstenhaber algebra, operads, homology, combinatorics, and quantum field theory where they occur in the theory of renormalization of Connes and Kreimer. While tridendriform algebra were introduced later by Loday and Ronco in their study of polytopes and Koszul duality [43].

Hom-tridendriform algebras were introduced in [48] as a twisted generalization of tridendriform algebras. More precisely, a Hom-tridendriform algebra is a linear space $A$, together with three bilinear maps $\dashv, \vdash, \cdot: A \otimes A \rightarrow A$ and a linear map $\alpha: A \rightarrow A$ satisfying, for $x, y, z \in A$,

$$
\begin{aligned}
(x \dashv y) \dashv \alpha(z) & =\alpha(x) \dashv(y \dashv z+y \vdash z+y \cdot z), \\
\alpha(x) \vdash(y \vdash z) & =(x \dashv y+x \vdash y+x \cdot y) \vdash \alpha(z), \\
(x \dashv y) \cdot \alpha(z) & =\alpha(x) \cdot(y \vdash z),(x \vdash y) \cdot \alpha(z)=\alpha(x) \vdash(y \cdot z), \\
(x \cdot y) \dashv \alpha(z) & =\alpha(x) \cdot(y \dashv z),(x \cdot y) \cdot \alpha(z)=\alpha(x) \cdot(y \cdot z), \\
(x \vdash y) \dashv \alpha(z) & =\alpha(x) \vdash(y \dashv z) .
\end{aligned}
$$

When $\alpha=i d$, we recover tridendriform algebras. The constructions of these algebras and their relationships with Hom-pre-Lie algebras are provided.

Connections between Hom-bialgebras and Hom-coalgebras, Hom-dendiform and tridendiform systems and Rota-Baxter and Hom-Rota-Baxter Hom-algebra structures are considered in [44-48,51]. 
As for Hom-Post-Lie algebras, they has been studied in [13] as a twisted generalization post-Lie algebra. Post-Lie algebras first arise from the work of Bruno Vallette [57] in 2007 through the purely operadic technique of Koszul dualization. In [52], it shown that they also arise naturally from differential geometry of homogeneous spaces and Klein geometries, topics that are closely related to Cartan's method of moving frames. The universal enveloping algebras of post-Lie algebras and the free post-Lie algebra are studied. Some examples and related structures are given. Further, they were extended to graded case in [16].

It has been noticed by D. Yau that one can deform associative and Lie algebra structures into Hom-associative and Hom-Lie algebras by composition of associative algebra multiplication with algebra endomorphism. This procedure, known as Yau's twisting or a composition method has been intensively used for many other Hom-algebraic structures such as Hom-Akivis algebras [17], Hom-Lie Yamagutti algebras [28], Hom-Poisson algebras [61], Hom-Post-Lie algebras [13], Hom-pre-Lie (or left Hom-symmetric) algebras [48,49], G-Hom-associative algebras [49,59], Hom-dendriform algebras [48,59], modules over Hom-bialgebras [62]. Left-Hom-symmetric dialgebras and Hom-Poisson dialgebras, their connection to Hom-Leibniz algebras using Loday commutator and their constructions using composition method have been considered in [15].

Here we prove that this technique works for a big class of Hom-algebraic structures and for other special linear maps as averaging operator or element of centroid. In the literature, the graded version, including super and color case, of many of these structures were studied.

The paper is organized as follows. In Sect. 2, we show on the one hand that the tensor product of two Hom-associative color algebra is also a Hom-associative color algebra. On the other hand, that the tensor product of a commutative Hom-associative color algebra and a Hom-left-symmetric color algebra is a Hom-left-symmetric color algebra. We give a construction of a Hom-associative color dialgebra from an associative color algebra and an averaging operator. Next, we introduce Hom-left-symmetric color dialgebras and study their connection with Hom-Poisson color dialgebras. In Sect. 3, we show that Hom-PostLie and Hom-associative color algebras may come from Hom-tridendriform color algebras. Next, we pointed out that the opposite of any Hom-tridendriform color algebra is also one, and any Hom-tridendriform color algebra carries a structure of Hom-dendriform algebra. Section 4 is devoted to a Yau's twisting generalization to Hom-algebraic structures with a finite number of bilinear products, and use averaging operators or centroids to produce other color Hom-algebras from given one.

Throughout this paper, all graded vector spaces are assumed to be over a field $\mathbb{K}$ of characteristic different from 2.

\section{Hom-left-symmetric color dialgebras}

For an abelian group $G$ and $G$-graded linear space $V=\bigoplus_{g \in G} V_{g}$, an element $x \in V$ is said to be homogeneous of the degree (color) $g \in G$ if $x \in V_{g}$. The set of all homogeneous elements of $V$ is denoted by $\mathcal{H}(V)$. A linear mapping $f: V \longrightarrow W$ between two $G$-graded linear spaces $V=\bigoplus_{g \in G} V_{g}$ and $W=\bigoplus_{g \in G} W_{g}$ is said to be homogeneous of the degree $d \in G$ if $f\left(V_{g}\right) \subseteq W_{g+d}$ for all $g \in G$. Homogeneous mapping is said to be even if it is of degree $0_{G}$ (degree zero), that is $f\left(V_{g}\right) \subseteq W_{g}$ for any $g \in G$. A bilinear mapping $f: V \times V \rightarrow V$ is said to be even if $f\left(V_{a}, V_{b}\right) \subseteq V_{a+b}$ for all $a, b \in G$. An algebra $\mathcal{A}$ is said to be $G$-graded if its underlying linear space is $G$-graded, that is $\mathcal{A}=\bigoplus_{g \in G} A_{g}$ and 
furthermore $A_{a} A_{b} \subseteq A_{a+b}$, for all $a, b \in G$. A homomorphism $f: A \longrightarrow B$ of $G$-graded algebras is a homomorphism of algebras which is also an even mapping.

Given an abelian group $G$, a map $\varepsilon: G \times G \rightarrow \mathbb{K}^{*}$ is called a skew-symmetric bicharacter or a commutation factor on $G$ if for all $a, b, c \in G$ :

(i) $\varepsilon(a, b) \varepsilon(b, a)=1$;

(ii) $\varepsilon(a, b+c)=\varepsilon(a, b) \varepsilon(a, c)$;

(iii) $\varepsilon(a+b, c)=\varepsilon(a, c) \varepsilon(b, c)$.

Remark that $\varepsilon(a, 0)=\varepsilon(0, a)=1, \varepsilon(a, a)= \pm 1$ for all $a \in G$, where 0 is the identity of $G$. If $x$ and $y$ are two homogeneous elements of degree $a$ and $b$ respectively and $\varepsilon$ is a skew-symmetric bicharacter, then we shorten the notation by writing $\varepsilon(x, y)$ instead of $\varepsilon(a, b)$.

Definition 1 [64] A Hom-associative color algebra is a triple $(A, \mu, \alpha)$ consisting of a $G$ graded linear space $A$, an even bilinear map $\mu: A \times A \rightarrow A$ and an even homomorphism $\alpha: A \rightarrow A$ satisfying

$$
\mu(\alpha(x), \mu(y, z))=\mu(\mu(x, y), \alpha(z)),
$$

for any $x, y, z \in \mathcal{H}(A)$ (homogeneous elements of $A$ ).

If in addition $\mu=\varepsilon(\cdot, \cdot) \mu^{o p}$ i.e. $\mu(x, y)=\varepsilon(x, y) \mu(y, x)$, for any $x, y \in \mathcal{H}(A)$, the color Hom-associative algebra $(A, \mu, \varepsilon, \alpha)$ is said to be a commutative Hom-associative color algebra.

Theorem 1 is proved by a direct calculation.

Theorem 1 Let $\left(A_{1}, \cdot 1, \varepsilon, \alpha_{1}\right)$ and $\left(A_{2}, \cdot 2, \varepsilon, \alpha_{2}\right)$ be two Hom-associative color algebras. Then $A=A_{1} \otimes A_{2}$ is a color Hom-associative algebra for twisting map $\alpha:=\alpha_{1} \otimes \alpha_{2}$ : $A \rightarrow A$ and the product $*: A \otimes A \rightarrow A$ defined by

$\alpha\left(a_{1} \otimes a_{2}\right):=\alpha_{1}\left(a_{1}\right) \otimes \alpha_{2}\left(a_{2}\right),\left(a_{1} \otimes a_{2}\right) *\left(b_{1} \otimes b_{2}\right):=\varepsilon\left(a_{2}, b_{1}\right)\left(a_{1} \cdot b_{1}\right) \otimes\left(a_{2} \cdot 2 b_{2}\right)$.

Corollary 1 The tensor product of two associative color algebras is also an associative color algebra.

Definition 2 A Hom-color algebra or a color Hom-algebra $(S, \cdot, \varepsilon, \alpha)$ is an $G$-graded linear space $S$ equipped with even bilinear multiplication ·, even twisting map $\alpha$ and commutation factor $\varepsilon$. A Hom-color algebra is called a Hom-left-symmetric color algebra if the following Hom-left-symmetric color identity (or $\varepsilon$-Hom-left-symmetric identity)

$$
(x \cdot y) \cdot \alpha(z)-\alpha(x) \cdot(y \cdot z)=\varepsilon(x, y)((y \cdot x) \cdot \alpha(z)-\alpha(y) \cdot(x \cdot z))
$$

is satisfied for all $x, y, z \in \mathcal{H}(S)$.

Example 1 Let $A=A_{(-1)} \oplus A_{(1)}=\left\langle e_{2}, e_{3}\right\rangle \oplus\left\langle e_{1}\right\rangle$ be a $G=\{-1,+1\}$-graded linear space. Then $A$ is a Hom-left-symmetric color algebra together with the bicharacter

$$
\varepsilon(i, j)=(-1)^{(i-1)(j-1) / 4},
$$

the multiplication $e_{1} e_{1}=-e_{1}, \quad e_{2} e_{1}=-a e_{2}, \quad e_{3} e_{1}=e_{3}, \quad e_{1} e_{2}=-a e_{2}$, and the even linear map $\alpha: A \rightarrow A$ defined by

$$
\alpha\left(e_{1}\right)=e_{1}, \quad \alpha\left(e_{2}\right)=a e_{2}, \quad \alpha\left(e_{3}\right)=-e_{3}, \quad a \in \mathbb{R} .
$$

Theorem 2 is proved by a straightforward computation. 
Theorem 2 If $\left(S, *, \varepsilon, \alpha_{S}\right)$ is a Hom-left-symmetric color algebra and $\left(A, \cdot, \varepsilon, \alpha_{A}\right)$ a commutative Hom-associative color algebra, then $\left(S \otimes A, \circ, \varepsilon, \alpha_{S \otimes A}\right)$ is a Hom-left-symmetric color algebra, with

$$
\begin{aligned}
\alpha_{S \otimes A} & =\alpha_{S} \otimes \alpha_{A}, \\
(x \otimes a) \circ(y \otimes b) & =\varepsilon(a, y)(x * y) \otimes(a \cdot b)
\end{aligned}
$$

for all $x, y \in \mathcal{H}(S), a, b \in \mathcal{H}(A)$.

Recall that an ideal of a color Hom-algebra $(A, \cdot, \varepsilon, \alpha)$ is a graded subspace $I$ such that $\alpha(I) \subseteq I$ and $I \cdot A=A \cdot I \subseteq I$.

Proposition 1 Let $(S, \cdot \varepsilon, \alpha)$ be a Hom-left-symmetric color algebra and I an ideal of $S$ such that for any $i, j \in \mathcal{H}(I)$ and $a, b, c, d \in \mathcal{H}(S)$,

$$
\begin{gathered}
\alpha(i) \cdot(a \cdot b)-(i \cdot a) \cdot \alpha(b)=\varepsilon(i, a)(\alpha(a) \cdot(i \cdot b)-(a \cdot i) \cdot \alpha(b)), \\
\alpha(c) \cdot(d \cdot j)-(c \cdot d) \cdot \alpha(j)=\varepsilon(c, d)(\alpha(d) \cdot(c \cdot j)-(d \cdot c) \cdot \alpha(j)) .
\end{gathered}
$$

Then $\left(I \oplus S, \dashv, \vdash, \alpha_{I \oplus S}\right)$ is a Hom-left-symmetric color dialgebra with

$$
\begin{aligned}
\alpha_{I \oplus S} & =\alpha_{I} \oplus \alpha_{S}, \\
\left(i_{1}+a_{1}\right) \dashv\left(i_{2}+a_{2}\right) & =i_{1} a_{2}+a_{1} a_{2}, \\
\left(i_{1}+a_{1}\right) \vdash\left(i_{2}+a_{2}\right) & =a_{1} i_{2}+a_{1} a_{2}
\end{aligned}
$$

for $i_{1}, a_{1} \in S_{p}, i_{1}, a_{2} \in S_{q}$.

Proof It is straightforward by calculation.

Definition 3 A Hom-left-symmetric color dialgebra is a $G$-graded linear space $S$ equipped with a bicharacter $\varepsilon: G \otimes G \rightarrow \mathbb{K}^{*}$ on $G$ and two even bilinear products $\dashv: S \times S \rightarrow S$ and $\vdash: S \times S \rightarrow S$ satisfying the identities

$$
\begin{aligned}
\alpha(x) \dashv(y \dashv z) & =\alpha(x) \dashv(y \vdash z), \\
(x \vdash y) \vdash \alpha(z) & =(x \dashv y) \vdash \alpha(z), \\
\alpha(x) \dashv(y \dashv z)-(x \dashv y) \dashv \alpha(z) & =\varepsilon(x, y)(\alpha(y) \vdash(x \dashv z)-(y \vdash x) \dashv \alpha(z)), \\
\alpha(x) \vdash(y \vdash z)-(x \vdash y) \vdash \alpha(z) & =\varepsilon(x, y)(\alpha(y) \vdash(x \vdash z)-(y \vdash x) \vdash \alpha(z))
\end{aligned}
$$

for all $x, y, z \in \mathcal{H}(S)$.

Remark 1 Relation (6) means that $(S, \vdash, \varepsilon, \alpha)$ is a Hom-left-symmetric color algebra. So, any Hom-left-symmetric color algebra is a Hom-left-symmetric color dialgebra.

Definition 4 [14] A Hom-associative color dialgebra is defined as a quintuple $(D, \dashv, \vdash, \varepsilon, \alpha)$ where $D$ is a $G$-graded linear space, $\dashv, \vdash: D \otimes D \rightarrow D$ are even bilinear maps, $\varepsilon: G \otimes G \rightarrow$ $\mathbb{K}^{*}$ is a bicharacter and $\alpha: D \rightarrow D$ is an even linear map such that the following five axioms

$$
\begin{aligned}
& (x \vdash y) \dashv \alpha(z)=\alpha(x) \vdash(y \dashv z), \\
& \alpha(x) \dashv(y \dashv z)=(x \dashv y) \dashv \alpha(z), \\
& (x \dashv y) \dashv \alpha(z)=\alpha(x) \dashv(y \vdash z), \\
& (x \vdash y) \vdash \alpha(z)=\alpha(x) \vdash(y \vdash z), \\
& \alpha(x) \vdash(y \vdash z)=(x \dashv y) \vdash \alpha(z)
\end{aligned}
$$

are satisfied for $x, y, z \in \mathcal{H}(D)$. 
Remark 2 If $(A, \dashv, \vdash, \varepsilon, \alpha)$ is a Hom-associative color dialgebra in which $\dashv=\vdash=: \mu$, then $(A, \mu, \varepsilon, \alpha)$ is a Hom-associative color algebra. Hence, any Hom-associative color algebra $(A, \mu, \varepsilon, \alpha)$ is a Hom-associative color dialgebra with $\dashv:=\mu=: \vdash$.

Lemma 1 A Hom-left-symmetric color dialgebra $S$ is a Hom-associative color dialgebra if and only if both products of $S$ are color Hom-associative.

Proof If a Hom-left-symmetric color dialgebra $S$ is a Hom-associative color dialgebra, then both products $\dashv$ and $\vdash$ defined over $S$ are Hom-associative according to Definition 4. Conversely, if each product of a Hom-left-symmetric color dialgebra is Hom-associative, then from (5), we get axiom (3).

We need the below definition for he next theorem.

Definition 5 A Nijenhuis operator on Hom-associative color algebra $(A, \mu, \varepsilon, \alpha)$ is an even linear map $N: A \rightarrow A$ such that $\alpha \circ N=N \circ \alpha$ and

$$
\mu(N(x), N(y))=N(\mu(N(x), y)+\mu(x, N(y))-N(\mu(x, y)))
$$

for all $x, y \in \mathcal{H}(A)$.

Theorem 3 Let $(A, \mu, \varepsilon, \alpha)$ be a Hom-associative color algebra and $N: A \rightarrow$ A a Nijenhuis operator. Then the new multiplication $\mu^{N}: A \rightarrow$ A given by

$$
\mu^{N}(x, y)=\mu(N(x), y)+\mu(x, N(y))-N(\mu(x, y))
$$

makes A into a Hom-associative color algebra.

Corollary 2 Let $(A, \mu, \varepsilon, \alpha)$ be an associative color algebra, $\alpha: A \rightarrow A$ be an even endomorphism and $N: A \rightarrow A$ be a Nijenhuis operator commuting with $\alpha$. Then $\mu_{\alpha}^{N}=\alpha \circ \mu^{N}$ is a Hom-associative color algebra.

Now we introduce averaging operator to produce Hom-associative color dialgebras from Hom-associative color algebras.

Definition 6 An averaging operator on Hom-associative color algebra $(A, \mu, \varepsilon, \alpha)$ is an even linear map $\beta: A \rightarrow A$ such that $\alpha \circ \beta=\beta \circ \alpha$ and

$$
\beta(\mu(\beta(x), y)=\mu(\beta(x), \beta(y))=\beta(\mu(x, \beta(y)))
$$

for all $x, y \in \mathcal{H}(A)$.

Theorem 4 Let $(A, \cdot, \varepsilon)$ be an associative color algebra and $\alpha: A \rightarrow A$ an averaging operator such that $(A, \cdot \varepsilon, \alpha)$ be a Hom-associative color algebra. For any $x, y \in \mathcal{H}(A)$, define new operations on $A$ by $x \vdash y=\alpha(x) \cdot y$ and $x \dashv y:=x \cdot \alpha(y)$. Then $(A, \dashv, \vdash, \varepsilon, \alpha)$ is a Hom-associative color dialgebra.

Proof We prove one axiom. The others are proved similarly. For all $x, y, z \in \mathcal{H}(A)$,

$$
\begin{aligned}
\alpha(x) \dashv(y \dashv z)-(x \dashv y) \dashv \alpha(z) & =\alpha(x) \cdot \alpha(y \cdot \alpha(z))-(x \cdot \alpha(y)) \cdot \alpha^{2}(z) \\
& \stackrel{(13)}{=} \alpha(x) \cdot(\alpha(y) \cdot \alpha(z))-(x \cdot \alpha(y)) \cdot \alpha^{2}(z) \\
& \stackrel{(1)}{=} \alpha(x) \cdot(\alpha(y) \cdot \alpha(z))-\alpha(x) \cdot(\alpha(y) \cdot \alpha(z))=0 .
\end{aligned}
$$

This proves the axiom. 
Definition 7 [14] A Hom-Poisson color dialgebra $(P, \dashv, \vdash,\{-,-\}, \varepsilon, \alpha)$ is a sextuple in which $P$ is a $G$-graded linear space, $\dashv, \vdash,\{-,-\}: P \otimes P \rightarrow P$ are three even bilinear maps, $\varepsilon: G \otimes G \rightarrow \mathbb{K}^{*}$ is a bicharacter and $\alpha: P \rightarrow P$ is an even linear map such that

$$
\begin{aligned}
& \{x \dashv y, \alpha(z)\}=\alpha(x) \dashv\{y, z\}+\varepsilon(y, z)\{x, z\} \dashv \alpha(y), \\
& \{x \vdash y, \alpha(z)\}=\alpha(x) \vdash\{y, z\}+\varepsilon(y, z)\{x, z\} \vdash \alpha(y), \\
& \{\alpha(x), y \dashv z\}=\varepsilon(x, y) \alpha(y) \vdash\{x, z\}+\{x, y\} \dashv \alpha(z)=\{\alpha(x), y \vdash z\}
\end{aligned}
$$

for all $x, y, z \in \mathcal{H}(P)$.

Lemma 2 [14] Let $(D, \dashv, \vdash, \varepsilon, \alpha)$ be a Hom-associative color dialgebra, and $\{x, y\}=x \dashv$ $y-\varepsilon(x, y) y \vdash x$ for any $x, y \in \mathcal{H}(D)$. Then $(D, \dashv, \vdash,\{-,-\}, \varepsilon, \alpha)$ is a Hom-Poisson color dialgebra.

Theorem 5 Let $(D, \dashv, \vdash, \varepsilon, \alpha)$ be a Hom-left-symmetric color dialgebra with Homassociative products. Then $(D, \dashv, \vdash,\{-,-\}, \varepsilon, \alpha)$ is a Hom-Poisson color dialgebra, where $\{x, y\}=x \dashv y-\varepsilon(x, y) y \vdash x$, for any $x, y \in \mathcal{H}(D)$.

Proof It follows from Lemmas 1 and 2.

\section{Hom-tridendriform color algebras}

We introduce Hom-tridendriform color algebras, give some properties and study their connection with Hom-post-Lie color algebras and color Hom-associative algebras.

Definition 8 A Hom-tridendriform color algebra is a sextuple $(T, \dashv, \vdash, \cdot, \varepsilon, \alpha)$ consisting of a $G$-graded linear space $T$, three even bilinear maps $\dashv, \vdash, \cdot: T \otimes T \rightarrow T$, a bicharacter $\varepsilon: G \times G \rightarrow \mathbb{K}^{*}$ and an even linear map $\alpha: T \rightarrow T$ satisfying

$$
\begin{aligned}
(x \dashv y) \dashv \alpha(z) & =\alpha(x) \dashv(y \dashv z+\varepsilon(z, y) y \vdash z+\varepsilon(z, y) y \cdot z), \\
(x \vdash y) \dashv \alpha(z) & =\varepsilon(z, x) \alpha(x) \vdash(y \dashv z), \\
\alpha(x) \vdash(y \vdash z) & =(\varepsilon(x, y) x \dashv y+x \vdash y+x \cdot y) \vdash \alpha(z), \\
(x \dashv y) \cdot \alpha(z) & =\varepsilon(y, x) \alpha(x) \cdot(y \vdash z), \\
(x \vdash y) \cdot \alpha(z) & =\alpha(x) \vdash(y \cdot z), \\
(x \cdot y) \dashv \alpha(z) & =\varepsilon(z, x) \alpha(x) \cdot(y \dashv z), \\
(x \cdot y) \cdot \alpha(z) & =\alpha(x) \cdot(y \cdot z)
\end{aligned}
$$

for $x, y, z \in \mathcal{H}(T)$.

Remark 3 When the color Hom-associative product is identically null, we get a Homdendriform color algebra [14].

Example 2 Let $A=A_{0} \oplus A_{1}=\left\langle e_{1}, e_{2}\right\rangle \oplus\left\langle e_{3}\right\rangle$ be a three-dimensional superspace. The multiplications

$$
\begin{array}{ll}
e_{2} \dashv e_{2}=e_{2} \vdash e_{2}=a e_{1}, & e_{2} \cdot e_{2}=-a e_{1}, \\
e_{3} \dashv e_{3}=e_{3} \vdash e_{3}=b e_{1}, & e_{3} \cdot e_{3}=b e_{1},
\end{array}
$$

and the even linear map $\alpha: A \rightarrow A$ defined by

$$
\alpha\left(e_{1}\right)=e_{1}, \quad \alpha\left(e_{2}\right)=e_{1}+e_{2}, \quad \alpha\left(e_{3}\right)=-e_{3}
$$

make $A$ into a Hom-tridendriform color algebra, for any $a, b \in \mathbb{R}$. 
Proposition 2 Let $(T, \dashv, \vdash, \cdot, \varepsilon, \alpha)$ be a Hom-tridendriform color algebra.

Then $\left(T, \dashv^{o p}, \vdash^{o p},{ }^{o p}, \varepsilon, \alpha\right)$ is also a Hom-tridendriform color algebra, with

$$
x \dashv^{o p} y:=y \vdash x, \quad x \vdash^{o p} y:=y \dashv x, \quad x .^{o p} y:=y \cdot x
$$

for any $x, y \in \mathcal{H}(T)$.

Proof The proof is straightforward by calculation by using axioms in Definition 8 .

Proposition 3 Let $(A, \dashv, \vdash, \cdot, \varepsilon, \alpha)$ be a Hom-tridendriform color algebra. Then $\left(A, \dashv, \vdash^{\prime}, \varepsilon, \alpha\right)$ is a Hom-dendriform color algebra, where $x \vdash^{\prime} y:=x \vdash y+x \cdot y$ for any $x, y \in \mathcal{H}(T)$.

Proof It comes immediately from Definition 8.

We need the following definition for the next proposition.

Definition 9 Let $(A, \cdot, \varepsilon, \alpha)$ be a Hom-associative color algebra and $\lambda \in \mathbb{K}$. An even linear map $R: A \rightarrow A$ is called a Rota-Baxter operator of weight $\lambda$ on $A$ if it satisfies the identities

$$
\begin{aligned}
R \circ \alpha & =\alpha \circ R, \\
R(x) \cdot R(y) & =R(R(x) \cdot y+x \cdot R(y)+\lambda x \cdot y),
\end{aligned}
$$

for any $x, y \in \mathcal{H}(A)$.

Theorem 6 Let $(A, \cdot, \varepsilon, \alpha, R)$ be a Rota-Baxter Hom-associative color algebra of weight $\lambda$. Let us define three new operations $\dashv, \vdash$ and $*$ on $A$ by

$$
x \dashv y:=x \cdot R(y), \quad x \vdash y:=\varepsilon(x, y) R(x) \cdot y \text { and } x * y:=\lambda \varepsilon(x, y) x \cdot y .
$$

Then $(A, \dashv, \vdash, *, \varepsilon, \alpha)$ is a Hom-tridendriform color algebra.

Example 3 Let $G=\{-1,+1\}$ be an abelian group and $A=A_{(-1)} \oplus A_{(1)}=\left\langle e_{2}\right\rangle \oplus\left\langle e_{1}\right\rangle$ a $G$-graded two-dimensional linear space. The quintuple $(A, \cdot, \varepsilon, \alpha, R)$ is a Rota-Baxter Hom-associative color algebra of weight $\lambda$ with

- the multiplication, $e_{1} \cdot e_{1}=-e_{1}, \quad e_{1} \cdot e_{2}=e_{2}, \quad e_{2} \cdot e_{1}=e_{2}, \quad e_{2} \cdot e_{2}=e_{1}$,

- the bicharacter, $\varepsilon(i, j)=(-1)^{(i-1)(j-1) / 4}$,

- the even linear map $\alpha: A \rightarrow A$ defined by $: \alpha\left(e_{1}\right)=e_{1}, \quad \alpha\left(e_{2}\right)=-e_{2}$,

- the Rota-Baxter operator $R: A \rightarrow A$ given by $: R\left(e_{1}\right)=-\lambda e_{1}, R\left(e_{2}\right)=-\lambda e_{2}$.

Therefore, $(A, \dashv, \vdash, *, \varepsilon, \alpha)$ is a Hom-tridendriform color algebra with

$$
\begin{aligned}
& e_{1} \dashv e_{1}=\lambda e_{1}, \quad e_{1} \vdash e_{1}=\lambda e_{1} \quad e_{1} * e_{1}=-\lambda e_{1}, \\
& e_{1} \dashv e_{2}=-\lambda e_{2}, e_{1} \vdash e_{2}=-\lambda e_{2}, e_{1} * e_{2}=\lambda e_{2}, \\
& e_{2} \dashv e_{1}=-\lambda e_{2}, e_{2} \vdash e_{1}=-\lambda e_{2} \quad e_{2} * e_{1}=\lambda e_{2}, \\
& e_{2} \dashv e_{2}=-\lambda e_{1}, e_{2} \vdash e_{2}=\lambda e_{1}, \quad e_{2} * e_{2}=-\lambda e_{1} .
\end{aligned}
$$

Proposition 4 Let $(A, \cdot, \varepsilon, R, \alpha)$ be a Rota-Baxter Hom-associative color algebra of weight $\lambda$. Define the operations $\dashv$ and $\vdash$ by

$$
x \dashv y:=x \cdot R(y)+\lambda x \cdot y \text { and } x \vdash y:=\varepsilon(x, y) R(x) \cdot y .
$$

Then $(A, \dashv, \vdash, \varepsilon)$ is a Hom-dendriform color algebra. 
Corollary 3 Let $(A, \cdot, \varepsilon, R)$ be a Rota-Baxter Hom-associative color algebra of weight 0. We define the even bilinear operations $\dashv: A \times A \rightarrow A$ and $\vdash: A \times A \rightarrow A$ on $A$ by $x \dashv y:=x \cdot R(y)$ and $x \vdash y:=\varepsilon(x, y) R(x) \cdot y$. Then $(A, \dashv, \vdash, \varepsilon)$ is a Hom-dendriform color algebra.

In Theorem 7, we associate a Hom-associative color algebra to any Hom-tridendriform color algebra.

Theorem 7 Let $(T, \dashv, \vdash, \cdot, \varepsilon, \alpha)$ be a Hom-tridendriform color algebra and define operation * by $x * y=x \vdash y+\varepsilon(x, y) x \dashv y+x \cdot y$. Then $(T, *, \varepsilon, \alpha)$ is a Hom-associative color algebra.

Proof For any $x, y, z \in \mathcal{H}(T)$

$$
\begin{aligned}
a s_{\alpha}(x, y, z)= & (x \vdash y) \vdash \alpha(z)+\varepsilon(x, y)(x \dashv y) \vdash \alpha(z)+(x \cdot y) \vdash \alpha(z) \\
& +\varepsilon(x, z) \varepsilon(y, z)(x \vdash y) \dashv \alpha(z)+\varepsilon(x, z) \varepsilon(y, z) \varepsilon(x, y)(x \dashv y) \dashv \alpha(z) \\
& +\varepsilon(x, z) \varepsilon(y, z)(x \cdot y) \dashv \alpha(z)+(x \vdash y) \cdot \alpha(z) \\
& +\varepsilon(x, y)(x \dashv y) \cdot \alpha(z)+(x \cdot y) \cdot \alpha(z)-\alpha(x) \vdash(y \vdash z) \\
& -\varepsilon(y, z) \alpha(x) \vdash(y \dashv z)-\alpha(x) \vdash(y \cdot z) \\
& -\varepsilon(x, y) \varepsilon(x, z) \alpha(x) \dashv(y \vdash z)-\varepsilon(x, y) \varepsilon(x, z) \varepsilon(y, z) \alpha(x) \dashv(y \dashv z) \\
& -\varepsilon(x, y) \varepsilon(x, z) \alpha(x) \dashv(y \cdot z)-\alpha(x) \cdot(y \vdash z) \\
& -\varepsilon(y, z) \alpha(x) \cdot(y \dashv z)-\alpha(x) \cdot(y \cdot z) .
\end{aligned}
$$

The left-hand side vanishes by axioms in Definition 8 . This prove that $(A, *, \varepsilon, \alpha)$ is a Homassociative color algebra. This completes the proof.

Remark 4 Whenever $(T, \dashv, \vdash, \cdot, \varepsilon, \alpha)$ is commutative, we recover [16, Lemma 3.3].

Definition 10 [12] A Hom-Poisson color algebra consists of a $G$-graded linear space $A$, a multiplication $\mu: A \times A \rightarrow A$, an even bilinear bracket $\{\cdot, \cdot\}: A \times A \rightarrow A$ and an even linear map $\alpha: A \rightarrow A$ such that

(i) $(A, \mu, \varepsilon, \alpha)$ is a Hom-associative color algebra,

(ii) $(A,\{\cdot, \cdot\}, \varepsilon, \alpha)$ is a Hom-Lie color algebra,

(iii) the color Hom-Leibniz identity is satisfied, i.e.,

$$
\{\alpha(x), \mu(y, z)\}=\mu(\{x, y\}, \alpha(z))+\varepsilon(x, y) \mu(\alpha(y),\{x, z\})
$$

for any $x, y, z \in \mathcal{H}(A)$.

Lemma 3 [12] Let $(A, \mu, \varepsilon, \alpha)$ be a Hom-associative color algebra.

Then $\left(A, \mu,\{\cdot, \cdot\}=\mu-\varepsilon(\cdot, \cdot) \mu^{o p}, \varepsilon, \alpha\right)$ is a Hom-Poisson color algebra.

Theorem 8 Let $(A, \dashv, \vdash, \cdot, \varepsilon, \alpha)$ be a Hom-tridendriform color algebra.

Then $(A, *,[-,-], \varepsilon, \alpha)$ is a Hom-Poisson color algebra, where

$$
x * y:=x \vdash y+\varepsilon(x, y) x \dashv y+x \cdot y \text { and }[x, y]:=x * y-\varepsilon(x, y) y * x .
$$

Proof The proof follows from Theorem 7 and Lemma 3. 
Definition 11 [16] A Hom-post-Lie color algebra $(L,[-,-], \cdot, \varepsilon, \alpha)$ is a Hom-Lie color algebra $(L,[-,-], \varepsilon, \alpha)$, i.e.

$$
\begin{aligned}
& {[x, y]=-\varepsilon(x, y)[y, x] \quad(\varepsilon \text {-skew-symmetry })} \\
& \varepsilon(z, x)[\alpha(x),[y, z]]+\varepsilon(x, y)[\alpha(y),[z, x]]+\varepsilon(y, z)[\alpha(z),[x, y]]=0 \\
& \quad(\varepsilon \text {-Hom-Jacobi identity) }
\end{aligned}
$$

together with an even bilinear map $\cdot: L \otimes L \rightarrow L$ such that

$$
\begin{aligned}
& \alpha(z) \cdot[x, y]-[z \cdot x, \alpha(y)]-\varepsilon(z, x)[\alpha(x), z \cdot y]=0, \\
& \alpha(z) \cdot(y \cdot x)-\varepsilon(z, y) \alpha(y) \cdot(z \cdot x)+\varepsilon(z, y)(y \cdot z) \cdot \alpha(x)-(z \cdot y) \cdot \alpha(x) \\
& \quad+\varepsilon(z, y)[y, z] \cdot \alpha(x)=0
\end{aligned}
$$

for any $x, y, z \in \mathcal{H}(L)$.

Example 4 Let $G=\mathbb{Z}_{2} \times \mathbb{Z}_{2}$ be an abelian group and $L$ be a three-dimensional $G$-graded linear space defined by

$$
L_{(0,0)}=0, \quad L_{(0,1)}=\left\langle e_{2}\right\rangle, \quad L_{(1,0)}=\left\langle e_{1}\right\rangle, \quad L_{(1,1)}=\left\langle e_{3}\right\rangle .
$$

Then $(L,[-,-], \cdot, \varepsilon, \alpha)$ is a Hom-Post-Lie color algebra with

- the bicharacter: $\varepsilon\left(\left(i_{1}, i_{2}\right),\left(j_{1}, j_{2}\right)\right)=(-1)^{i_{1} j_{1}+i_{2} j_{2}}$,

- the bracket: $\left[e_{1}, e_{2}\right]=-e_{3},\left[e_{1}, e_{3}\right]=-e_{2}, \quad\left[e_{2}, e_{3}\right]=-e_{1}$,

- the multiplication: $e_{2} e_{1}=-e_{3}, e_{2} e_{3}=e_{1}$,

- the even linear map $\alpha: L \rightarrow L: \alpha\left(e_{1}\right)=-e_{1}, \alpha\left(e_{2}\right)=-e_{2}, \alpha\left(e_{3}\right)=e_{3}$.

Proposition 5 Let $(L,[-,-], \cdot, \varepsilon, \alpha)$ be a color post-Hom-Lie algebra. With the product $x * y=x \cdot y+\frac{1}{2}[x, y],(L, *, \varepsilon, \alpha)$ is a color Hom-Lie admissible algebra, that is the map $*: L \times L \rightarrow L$ satisfies the $\varepsilon$-Hom-Jacobi identity.

Proof It follows from direct computation by using axioms in Definition 11.

To any Hom-tridendriform color algebra one can associate a Hom-post-Lie color algebra, as stated in the following result.

Theorem 9 Let $(T, \dashv, \vdash, \cdot, \varepsilon, \alpha)$ be a Hom-tridendriform color algebra, and let $x \circ y=x \vdash$ $y-y \dashv x$ and $[x, y]=x \cdot y-\varepsilon(x, y) y \cdot x$ for any $x, y \in \mathcal{H}(T)$. Then $(T, \circ,[-,-], \varepsilon, \alpha)$ is a Hom-post-Lie color algebra.

Proof The $\varepsilon$-skew-symmetry and the $\varepsilon$-Hom-Jacobi identity are trivial, by Lemma 3 . Now, for any $x, y, z \in \mathcal{H}(T)$, we have

$$
\begin{aligned}
& \alpha(x) \circ(y \circ z)-\varepsilon(x, y) \alpha(y) \circ(x \circ z)+\varepsilon(x, y)(y \circ x) \circ \alpha(z)-(x \circ y) \circ \alpha(z) \\
& \quad+\varepsilon(x, y)[y, x] \circ \alpha(z)=\alpha(x) \vdash(y \vdash z)-\alpha(x) \vdash(z \dashv y)-(y \vdash z) \dashv \alpha(x) \\
& \quad+(z \dashv y) \dashv \alpha(x)-\varepsilon(x, y) \alpha(y) \vdash(x \vdash z) \\
& \quad+\varepsilon(x, y) \alpha(y) \vdash(z \dashv x)+\varepsilon(x, y)(x \vdash z) \dashv \alpha(y) \\
& \quad-\varepsilon(x, y)(z \dashv x) \dashv \alpha(y)+\varepsilon(x, y)(y \vdash x) \vdash \alpha(z) \\
& \quad-\varepsilon(x, y)(x \dashv y) \vdash \alpha(z) \\
& \quad-\varepsilon(x, y) \alpha(z) \dashv(y \vdash x) \\
& \quad+\varepsilon(x, y) \alpha(z) \dashv(x \dashv y)-(x \vdash y) \vdash \alpha(z)+(y \dashv x) \vdash \alpha(z)
\end{aligned}
$$




$$
\begin{aligned}
& +\alpha(z) \dashv(x \vdash y)-\alpha(z) \dashv(y \dashv x) \\
& +\varepsilon(x, y)(y \cdot x) \vdash \alpha(z)-(x \cdot y) \vdash \alpha(z)-\varepsilon(x, y) \alpha(z) \dashv(y \cdot x)+\alpha(z) \dashv(x \cdot y)
\end{aligned}
$$

which vanishes by axioms in Definition 8 . Next,

$$
\begin{aligned}
& \alpha(z) \circ[x, y]-[z \circ x, \alpha(y)]-\varepsilon(z, x)[\alpha(x), z \circ y] \\
& =\alpha(z) \vdash(x \cdot y)-\varepsilon(x, y) \alpha(z) \vdash(y \cdot x)-(x \cdot y) \dashv \alpha(z)+\varepsilon(x, y)(y \cdot x) \dashv \alpha(z) \\
& \quad-(z \vdash x) \cdot \alpha(y)+(x \dashv z) \cdot \alpha(y)+\varepsilon(x+z, y) \alpha(y) \cdot(z \vdash x) \\
& \quad-\varepsilon(x+z, y) \alpha(y) \cdot(x \dashv z)-\varepsilon(z, x) \alpha(x) \cdot(z \vdash y)+\varepsilon(z, x) \alpha(x) \cdot(y \dashv z) \\
& \quad+\varepsilon(x, y)(z \vdash y) \cdot \alpha(x)-\varepsilon(x, y)(y \dashv z) \cdot \alpha(x) .
\end{aligned}
$$

The left-hand side vanishes by axioms in Definition 8 .

\section{Generalization of Yau's twisting composition method}

In this section, we generalize Yau's twisting to a large class of color Hom-algebras and use the centroids to produce other color Hom-algebras from given one. To state the fundamental result, Theorem 10, of this section, we give the following definitions.

Definition 12 By a color Hom-algebra we mean a $(n+3)$-uple $\left(A, \mu_{1}, \ldots, \mu_{n}, \varepsilon, \alpha\right)$ in which $A$ is a $G$-graded linear space, $\mu_{i}: A \otimes A \rightarrow A(i=1, \ldots, n)$ are even bilinear maps, $\varepsilon: G \times G \rightarrow \mathbb{K}^{*}$ is a bicharacter and $\alpha: A \rightarrow A$ is an even linear map, called the twisting map. If in addition, $\alpha \circ \mu_{i}=\mu_{i} \circ(\alpha \otimes \alpha)(i=1, \ldots, n)$, the color Hom-algebra $\left(A, \mu_{1}, \ldots, \mu_{n}, \varepsilon, \alpha\right)$ is said to be multiplicative. We call a Hom- $X$ color algebra a color Hom-algebra for which the axioms defining the structure of $X$ are linear combination of the terms of the form $\mu_{j} \circ\left(\mu_{i} \otimes \alpha\right)$ or $\mu_{j} \circ\left(\alpha \otimes \mu_{i}\right)$.

Example 5 (1) A Hom- $X$ color algebra $(A, \mu, \varepsilon, \alpha)$ for which

$$
\mu(\mu(x, y), \alpha(z))-\mu(\alpha(x), \mu(y, z))=0
$$

is called a Hom-associative color algebra.

(2) A Hom- $X$ color algebra $(A, \mu, \varepsilon, \alpha)$ for which

$$
\mu(\alpha(x), \mu(y, z))+\varepsilon(y, z) \mu(\mu(x, z), \alpha(y))-\mu(\mu(x, y), \alpha(z))=0
$$

is called a Hom-Leibniz color algebra.

(3) A Hom- $X$ color algebra $\left(A, \mu_{1}, \mu_{2}, \varepsilon, \alpha\right)$ for which (31) and (32) hold for $\mu_{1}$ and $\mu_{2}$ respectively and

$$
\mu_{2}\left(\alpha(x), \mu_{1}(y, z)\right)+\varepsilon(y, z) \mu_{2}\left(\mu_{1}(x, z), \alpha(y)\right)-\mu_{1}\left(\mu_{2}(x, y), \alpha(z)=0\right.
$$

is called a Hom-Leibniz-Poisson color algebra.

Remark 5 The following color Hom-algebras enter in these categories of algebras: HomLie color algebras, Hom-pre-Lie (or left Hom-symmetric) color algebras, Hom-post-Lie color algebras, Hom-left symmetric color dialgebras, Hom-Poisson color dialgebras, Hompre-Poisson color algebras, Hom-post-Poisson color algebras, Hom-Leibniz-Poisson color algebras, Hom-tridendriform color algebras and so on. 
Definition 13 Let $\left(A, \mu_{1}, \ldots, \mu_{n}, \varepsilon, \alpha\right)$ and $\left(A^{\prime}, \mu_{1}^{\prime}, \ldots, \mu_{n}^{\prime}, \varepsilon, \alpha^{\prime}\right)$ be two color Homalgebras. An even linear map $f: A \rightarrow A^{\prime}$ is said to be a morphism of color Hom-algebras if $f \circ \alpha=\alpha^{\prime} \circ f$ and $f\left(\mu_{i}(x, y)\right)=\mu_{i}^{\prime}(f(x), f(y))$ for all $x, y \in \mathcal{H}(A)$ and $i=1, \ldots, n$.

Definition 14 Let $\left(A, \mu_{1}, \ldots, \mu_{n}, \varepsilon, \alpha\right)$ be a multiplicative color Hom-algebra.

(1) For $k \in \mathbb{N}^{*}=\{1,2,3, \ldots\}$, the $k t h$ derived color Hom-algebra of type 1 of $A$ is defined by

$$
A_{1}^{k}=\left(A, \mu_{1}^{(k)}=\alpha^{k} \circ \mu_{1}, \ldots, \mu_{n}^{(k)}=\alpha^{k} \circ \mu_{n}, \varepsilon, \alpha^{k+1}\right) .
$$

(2) For $k \in \mathbb{N}^{*}=\{1,2,3, \ldots\}$, the $k t h$ derived color Hom-algebra of type 2 of $A$ is defined by

$$
A_{2}^{k}=\left(A, \mu_{1}^{\left(2^{k}-1\right)}=\alpha^{2^{k}-1} \circ \mu_{1}, \ldots, \mu_{n}^{\left(2^{k}-1\right)}=\alpha^{2^{k}-1} \circ \mu_{n}, \varepsilon, \alpha^{2^{k}}\right)
$$

and $A_{1}^{0}=A_{2}^{0}=\left(A, \mu_{1}, \ldots, \mu_{n}, \varepsilon, \alpha\right)$ and $A_{1}^{1}=A_{2}^{1}=\left(A, \alpha \circ \mu_{1}, \ldots, \alpha \circ \mu_{n}, \varepsilon, \alpha^{2}\right)$.

Definition 15 A color Hom-algebra $\left(A, \mu_{1}, \ldots, \mu_{n}, \varepsilon, \alpha\right)$ endowed with an even linear map $R: A \rightarrow A$ such that

$$
\mu_{i}(R(x), R(y))=R\left(\mu_{i}(R(x), y)+\mu_{i}(x, R(y))+\lambda \mu_{i}(x, y)\right), \quad i=1, \ldots, n
$$

with $\lambda \in \mathbb{K}$ and $x, y \in \mathcal{H}(A)$, is called a Rota-Baxter color Hom-algebra, and $R$ is called a Rota-Baxter operator on $A$.

The below result allows to get Hom- $X$ color algebras from either an $X$-color algebra on the one hand or another Hom- $X$ color algebra on the other hand.

Theorem 10 Let $\left(A, \mu_{1}, \ldots, \mu_{n}, \varepsilon, \alpha\right)$ be a Rota-Baxter Hom-X color algebra and $\beta: A \rightarrow$ $A$ be an endomorphism of $A$. Then, for any nonnegative integer $n$,

$$
A_{\beta}=\left(A, \mu_{\beta}^{1}=\beta^{n} \circ \mu_{1}, \ldots, \mu_{\beta}^{n}=\beta^{n} \circ \mu_{n}, \beta^{n} \circ \alpha\right)
$$

is a Rota-Baxter Hom-X color algebra, where $\beta^{n}=\beta \circ \beta^{n-1}$.

Moreover, suppose that $\left(A^{\prime}, \mu_{1}^{\prime}, \ldots, \mu_{n}^{\prime}, \varepsilon, \alpha^{\prime}\right)$ is another Hom-X color algebra and $\beta^{\prime}$ : $A^{\prime} \rightarrow A^{\prime}$ be an algebra endomorphism. If $f: A \rightarrow A^{\prime}$ is a morphism of Hom-X color algebras that satisfies $f \circ \beta=\beta^{\prime} \circ f$, then $f: A_{\beta} \rightarrow A_{\beta^{\prime}}^{\prime}$ is also a morphism of Hom-X color algebras.

Proof The proof of the first part follows from the following facts.

For any $x, y, z \in \mathcal{H}(X), 1 \leq i, j \leq n$,

$$
\begin{aligned}
\mu_{\beta}^{i}\left(\mu_{\beta}^{j}(x, y),\left(\beta^{n} \circ \alpha\right)(z)\right) & =\mu_{\beta}^{i}\left(\mu_{\beta}^{j}(x, y), \beta^{n}(\alpha(z))\right) \\
& =\beta^{n} \mu_{i}\left(\beta^{n} \mu_{j}(x, y), \beta^{n}(\alpha(z))\right) \\
& =\left(\beta^{n} \circ \beta^{n}\right)\left(\mu_{i}\left(\mu_{j}(x, y), \alpha(z)\right)\right) \\
& =\beta^{2 n}\left(\mu_{i}\left(\mu_{j}(x, y), \alpha(z)\right)\right), \\
\mu_{\beta}^{i}\left(\left(\beta^{n} \circ \alpha\right)(x), \mu_{\beta}^{j}(y, z)\right) & =\mu_{\beta}^{i}\left(\beta^{n}(\alpha(x)), \mu_{\beta}^{j}(y, z)\right) \\
& =\beta^{n}\left(\mu_{i}\left(\beta^{n}(\alpha(x)), \beta^{n}\left(\mu_{j}(y, z)\right)\right)\right. \\
& =\left(\beta^{n} \circ \beta^{n}\right)\left(\mu_{i}\left(\alpha(x), \mu_{j}(y, z)\right)\right)
\end{aligned}
$$




$$
=\beta^{2 n}\left(\mu_{i}\left(\alpha(x), \mu_{j}(y, z)\right)\right) .
$$

To prove the Rota-Baxter identity (36) for $\mu_{\beta}^{i}$, we have

$$
\begin{aligned}
\mu_{\beta}^{i}(x, y) & =\beta^{n}\left(\mu_{i}(x, y)\right)=\beta^{n}\left(\mu_{i}(R(x), y)+\mu_{i}(x, R(y))+\lambda \mu_{i}(x, y)\right) \\
& =\beta^{n} \mu_{i}(R(x), y)+\beta^{n} \mu_{i}(x, R(y))+\lambda \beta^{n} \mu_{i}(x, y) \\
& =\mu_{\beta}^{i}(R(x), y)+\mu_{\beta}^{i}(x, R(y))+\lambda \mu_{\beta}^{i}(x, y) .
\end{aligned}
$$

For the second assertion, we have

$$
\begin{aligned}
f\left(\mu_{\beta}^{i}(x, y)\right) & =f\left(\beta^{n}\left(\mu_{i}(x, y)\right)\right)=f\left(\mu_{i}\left(\beta^{n}(x), \beta^{n}(y)\right)=\mu_{i}^{\prime}\left(f\left(\beta^{n}(x)\right), f\left(\beta^{n}(y)\right)\right.\right. \\
& =\mu_{i}^{\prime}\left(\beta^{\prime n}(f(x)), \beta^{\prime n}(f(y))=\beta^{\prime n}\left(\mu_{i}^{\prime}(f(x), f(y))=\mu_{\beta}^{\prime i}(f(x), f(y)) .\right.\right.
\end{aligned}
$$

This ends the proof.

Remark 6 Whenever $\alpha=I d$ in the first part of the previous theorem, we obtain a Hom- $X$ color algebra from an $X$-color algebra.

Example 6 If $(T, \dashv, \vdash, \cdot \varepsilon, \alpha)$ is a Hom-tridendriform color algebra and $\beta: T \rightarrow T$ an endomorphism of $T$, then $T_{\beta}=\left(T, \dashv_{\beta}=\beta^{n} \circ \dashv, \vdash_{\beta}=\beta^{n} \circ \vdash, \cdot \beta=\beta^{n} \circ \cdot, \beta^{n} \circ \alpha\right)$ is a Hom-tridendriform color algebra, for any nonnegative integer $n$.

Moreover, suppose that $\left(T^{\prime}, \dashv^{\prime}, \vdash^{\prime}, .^{\prime}, \varepsilon, \alpha^{\prime}\right)$ is another Hom-tridendriform color algebra and $\beta^{\prime}: T^{\prime} \rightarrow T^{\prime}$ a Hom-tridendriform color algebra endomorphism. If $f: T \rightarrow T^{\prime}$ is a morphism of Hom-tridendriform color algebra that satisfies $f \circ \beta=\beta^{\prime} \circ f$, then $f: T_{\beta} \rightarrow T_{\beta^{\prime}}^{\prime}$ is a morphism of Hom-tridendriform color algebras.

Proof We shall only prove relation (17), the others being proved analogously. Then, for any $x, y, z \in \mathcal{H}(T)$,

$$
\begin{aligned}
\left(x \dashv_{\beta} y\right) \dashv_{\beta}\left(\beta^{n} \circ \alpha\right)(z) & =\beta^{n}\left(\beta^{n}(x \dashv y) \dashv\left(\beta^{n} \circ \alpha\right)(x)\right)=\beta^{2 n}((x \dashv y) \dashv \alpha(x)) \\
& =\beta^{2 n}(\alpha(x) \dashv(y \dashv z+\varepsilon(z, y) y \vdash z+\varepsilon(z, y) y \cdot z) \\
& =\left(\beta^{n} \circ \alpha\right)(x) \dashv_{\beta} \beta^{n}(y \dashv z+\varepsilon(z, y) y \vdash z+\varepsilon(z, y) y \cdot z) \\
& =\left(\beta^{n} \circ \alpha\right)(x) \dashv_{\beta}\left(y \dashv_{\beta} z+\varepsilon(z, y) y \vdash_{\beta} z+\varepsilon(z, y) y \cdot \beta z\right) .
\end{aligned}
$$

For the second assertion, we have

$$
\begin{aligned}
f\left(x \dashv_{\beta} y\right) & \left.\left.=f\left(\beta^{n}(x) \dashv \beta^{n}(y)\right)\right)=f\left(\beta^{n}(x)\right) \dashv^{\prime} f\left(\beta^{n}(y)\right)\right) \\
& =\beta^{\prime n}(f(x)) \dashv^{\prime} \beta^{\prime n}(f(y))=f(x) \dashv_{\beta^{\prime}}^{\prime} f(y) .
\end{aligned}
$$

This completes the proof.

We have the following series of consequence of Theorem 10.

Corollary 4 Let $\left(A, \mu_{1}, \ldots, \mu_{n}, \varepsilon\right)$ be an $X$-color algebra and $\beta: A \rightarrow A$ be an endomorphism of $A$. Then $A_{\beta}=\left(A, \beta \circ \mu_{1}, \ldots, \beta \circ \mu_{n}, \varepsilon, \beta\right)$ is a multiplicative Hom-X color algebra.

Proof Take $\alpha=I d$ in Theorem 10.

Corollary 5 Let $\left(A, \mu_{1}, \ldots, \mu_{n}, \varepsilon, \alpha\right)$ be a Hom-X color algebra such that $\alpha$ be invertible. Then $\left(A, \mu_{\alpha^{-1}}=\alpha^{-1} \circ \mu_{1}, \ldots, \mu_{\alpha^{-1}}=\alpha^{-1} \circ \mu_{n}, \varepsilon\right)$ is an $X$-color algebra.

Proof Take $n=1$ and $\beta=\alpha^{-1}$ in Theorem 10 . 
Corollary 6 Let $\left(A, \mu_{1}, \ldots, \mu_{n}, \varepsilon, \alpha\right)$ be a Hom-X color algebra. Then the kth derived color Hom-algebra of type 1 and the kth derived color Hom-algebra of type 2 are Hom-X color algebras.

Proof It is sufficient to take $\beta=\alpha$, and $n=k$ and $n=2^{k}-1$ respectively in Theorem 10 .

Now we introduce the notion of centroids for Hom- $X$ color algebras. They allow to provide Hom- $X$ color algebras from a given one, and thus they play here the role of twisting.

Definition 16 Let $\left(A, \mu_{1}, \ldots, \mu_{n}, \varepsilon, \alpha\right)$ be a color Hom-algebra. An even linear map $\beta$ : $A \rightarrow A$ is said to be an element of the centroid if

$$
\beta\left(\mu_{i}(x, y)\right)=\mu_{i}(\beta(x), y)=\mu_{i}(x, \beta(y)) \text { for any } x, y \in \mathcal{H}(A) .
$$

The centroid of $A$ is defined by

$$
\begin{gathered}
\operatorname{Cent}(A)=\{\beta: A \rightarrow A \text { even linear map } \mid \forall x, y \in \mathcal{H}(A): \\
\left.\beta\left(\mu_{i}(x, y)\right)=\mu_{i}(\beta(x), y)=\mu_{i}(x, \beta(y))\right\}
\end{gathered}
$$

Theorem 11 Let $\left(A, \mu_{1}, \ldots, \mu_{n}, \varepsilon, R, \alpha\right)$ be a Rota-Baxter Hom-X color algebra and $\beta_{1}, \beta_{2}: A \rightarrow A$ be a pair of commuting elements of the centroid such that $\beta_{i} \circ R=$ $R \circ \beta_{i}, i=1,2$.

(1) Define bilinear maps $\mu_{\beta}^{i}: A \times A \rightarrow A,(i=1, \ldots, n)$, by

$$
\mu_{\beta}^{i}(x, y):=\mu_{i}\left(\left(\beta_{2} \beta_{1}\right)(x), y\right) .
$$

Then $A_{\beta_{1}, \beta_{2}}^{1}=\left(A, \mu_{\beta}^{1}, \ldots, \mu_{\beta}^{n}, \varepsilon, R, \beta_{2} \beta_{1} \alpha\right)$ is also a Rota-Baxter Hom-X color algebra.

(2) Define bilinear maps $\mu_{\beta}^{i}: A \times A \rightarrow A,(i=1, \ldots, n)$, by

$$
\mu_{\beta}^{i}(x, y):=\mu_{i}\left(\beta_{1}(x), \beta_{2}(y)\right) .
$$

Then $A_{\beta_{1}, \beta_{2}}^{2}=\left(A, \mu_{\beta}^{1}, \ldots, \mu_{\beta}^{n}, \varepsilon, R, \beta_{2} \beta_{1} \alpha\right)$ is also a Rota-Baxter Hom-X color algebra.

Proof (1) For any $x, y \in \mathcal{H}(A)$, we have

$$
\begin{aligned}
\mu_{\beta}^{i}\left(\mu_{\beta}^{j}(x, y),\left(\beta_{2} \beta_{1} \alpha\right)(z)\right) & =\mu_{\beta}^{i}\left(\mu_{\beta}^{j}(x, y), \beta_{2}\left(\beta_{1}(\alpha(z))\right)\right) \\
& =\mu_{i}\left(\beta_{2} \beta_{1}\left(\mu_{j}\left(\beta_{2} \beta_{1}(x), y\right)\right), \beta_{2} \beta_{1}(\alpha(z))\right) \\
& =\beta_{2} \mu_{i}\left(\beta_{1}\left(\beta_{2} \mu_{j}\left(\beta_{1}(x), y\right)\right), \beta_{2} \beta_{1}(\alpha(z))\right) \\
& =\beta_{2}^{2} \mu_{i}\left(\beta_{1}\left(\beta_{2} \mu_{j}\left(\beta_{1}(x), y\right)\right), \beta_{1}(\alpha(z))\right) \\
& \left.=\beta_{2}^{2} \beta_{1} \mu_{i}\left(\beta_{2} \mu_{j}\left(\beta_{1}(x), y\right)\right), \beta_{1}(\alpha(z))\right) \\
& \left.=\beta_{2}^{3} \beta_{1} \mu_{i}\left(\beta_{1} \mu_{j}(x, y)\right), \beta_{1}(\alpha(z))\right) \\
& \left.=\beta_{2}^{3} \beta_{1}^{2} \mu_{i}\left(\mu_{j}(x, y)\right), \beta_{1}(\alpha(z))\right) \\
& =\beta_{2}^{3} \beta_{1}^{3} \mu_{i}\left(\mu_{j}(x, y), \alpha(z)\right) .
\end{aligned}
$$

We have a similar proof for $\mu_{\beta}^{i}\left(\left(\beta_{2} \beta_{1} \alpha\right)(x), \mu_{\beta}^{j}(y, z)\right)$. Next,

$$
\mu_{\beta}^{i}(R(x), R(y))=\mu_{i}\left(\beta_{2} \beta_{1}(R(x)), R(y)\right)=\beta_{2} \beta_{1} \mu_{i}(R(x), R(y))
$$




$$
\begin{aligned}
& =\beta_{2} \beta_{1} R\left(\mu_{i}(R(x), y)+\mu_{i}(x, R(y))+\lambda \mu_{i}(x, y)\right) \\
& =R\left(\beta_{2} \beta_{1} \mu_{i}(R(x), y)+\beta_{2} \beta_{1} \mu_{i}(x, R(y))+\lambda \beta_{2} \beta_{1} \mu_{i}(x, y)\right) \\
& =R\left(\mu_{i}\left(\beta_{2} \beta_{1} R(x), y\right)+\mu_{i}\left(\beta_{2} \beta_{1}(x), R(y)\right)+\lambda \mu_{i}\left(\beta_{2} \beta_{1}(x), y\right)\right) \\
& =R\left(\mu_{\beta}^{i}(R(x), y)+\mu_{\beta}^{i}(x, R(y))+\lambda \mu_{\beta}^{i}(x, y)\right) .
\end{aligned}
$$

(2) For any $x, y \in \mathcal{H}(A)$, we have

$$
\begin{aligned}
\mu_{\beta}^{i}\left(\mu_{\beta}^{j}(x, y),\left(\beta_{2} \beta_{1} \alpha\right)(z)\right) & =\mu_{\beta}^{i}\left(\mu_{\beta}^{j}(x, y), \beta_{2}\left(\beta_{1}(\alpha(z))\right)\right) \\
& =\mu_{i}\left(\beta_{1}\left(\mu_{j}\left(\beta_{1}(x), \beta_{2}(y)\right)\right), \beta_{2}\left(\beta_{2} \beta_{1}(\alpha(z))\right)\right) \\
& =\beta_{1} \beta_{2} \mu_{i}\left(\beta_{1} \beta_{2}\left(\mu_{j}(x, y)\right), \beta_{2} \beta_{1}(\alpha(z))\right) \\
& =\beta_{1}^{2} \beta_{2}^{2} \mu_{i}\left(\mu_{j}(x, y), \beta_{2} \beta_{1}(\alpha(z))\right), \\
& =\beta_{1}^{3} \beta_{2}^{3} \mu_{i}\left(\mu_{j}(x, y), \alpha(z)\right) .
\end{aligned}
$$

Similarly, one proves $\mu_{\alpha}^{i}\left(\left(\beta_{2} \beta_{1} \alpha\right)(x), \mu_{\alpha}^{j}(y, z)\right)=\beta_{1}^{3} \beta_{2}^{3} \mu_{i}\left(\alpha(x), \mu_{j}(y, z)\right)$. Next,

$$
\begin{aligned}
& \mu_{\beta}^{i}(R(x), R(y))=\mu_{i}\left(\beta_{1}(R(x)), \beta_{2} R(y)\right)=\beta_{2} \beta_{1} \mu_{i}(R(x), R(y)) \\
& \quad \beta_{2} \beta_{1} R\left(\mu_{i}(R(x), y)+\mu_{i}(x, R(y))+\lambda \mu_{i}(x, y)\right) \\
& =R\left(\beta_{2} \beta_{1} \mu_{i}(R(x), y)+\beta_{2} \beta_{1} \mu_{i}(x, R(y))+\lambda \beta_{2} \beta_{1} \mu_{i}(x, y)\right) \\
& =R\left(\mu_{i}\left(\beta_{1} R(x), \beta_{2}(y)\right)+\mu_{i}\left(\beta_{1} x, \beta_{2}(R(y))\right)+\lambda \mu_{i}\left(\beta_{1}(x), \beta_{2}(y)\right)\right) \\
& =R\left(\mu_{\beta}^{i}(R(x), y)+\mu_{\beta}^{i}(x, R(y))+\lambda \mu_{\beta}^{i}(x, y)\right) .
\end{aligned}
$$

This completes the proof.

Let us observe that $A_{\beta_{1}, I d}^{1}=A_{\beta_{1}, I d}^{2}$.

Corollary 7 Let $\left(A, \mu_{1}, \ldots, \mu_{n}, \varepsilon, R, \alpha\right)$ be a Rota-Baxter Hom-X color algebra and $\beta \in$ Cent $(A)$. Define bilinear maps $\mu_{\beta}^{i}: A \times A \rightarrow A,(i=1, \ldots, n)$, by

$$
\mu_{\beta}^{i}(x, y):=\mu_{i}(\beta(x), y) .
$$

Then $\left(A, \mu_{\beta}^{1}, \ldots, \mu_{\beta}^{n}, \varepsilon, R, \beta \circ \alpha\right)$ is also a Rota-Baxter Hom-X color algebra.

Note that $\left(A, \mu_{\beta}^{1}, \ldots, \mu_{\beta}^{n}, \varepsilon, \beta\right)$ is also a Hom- $X$ color algebra whenever $\alpha$ is the identity map in Corollary 7.

Acknowledgements Dr. Ibrahima Bakayoko is grateful to the research environment MAM in Mathematics and Applied Mathematics, Division of Mathematics and Physics, School of Education, Culture and Communication at Mälardalen University for hospitality and an excellent and inspiring environment for research and research education and cooperation in Mathematics during his visit aimed at broadening activities of research and research education capacity and cooperation development programmes in Mathematics in Africa supported by International Science Programme in Mathematics and The Swedish International Development Cooperation Agency.

Funding Open Access funding provided by Mälardalen University.

Open Access This article is licensed under a Creative Commons Attribution 4.0 International License, which permits use, sharing, adaptation, distribution and reproduction in any medium or format, as long as you give appropriate credit to the original author(s) and the source, provide a link to the Creative Commons licence, and indicate if changes were made. The images or other third party material in this article are included in the article's Creative Commons licence, unless indicated otherwise in a credit line to the material. If material is not included in the article's Creative Commons licence and your intended use is not permitted by statutory regulation or exceeds the permitted use, you will need to obtain permission directly from the copyright holder. To view a copy of this licence, visit http://creativecommons.org/licenses/by/4.0/. 


\section{References}

1. Abdaoui, K., Ammar, F., Makhlouf, A.: Constructions and cohomology of Hom-Lie color algebras. Commun. Algebra 43(11), 4581-4612 (2015)

2. Aizawa, N., Sato, H., $q$-Deformation of the Virasoro algebra with central extension. Phys. Lett. B 256, 185-190 (1991) (Hiroshima University preprint, preprint HUPD-9012 (1990))

3. Ammar, F., Ayadi, I., Mabrouk, S., Makhlouf, A.: Quadratic color Hom-Lie algebras, In: Siles Molina, M., El Kaoutit, L., Louzari, M., Ben Yakoub, L., Benslimane, M. (eds.) Associative and Non-Associative Algebras and Applications. MAMAA 2018. Springer Proceedings in Mathematics and Statistics, vol. 311. Springer, Cham, pp. 287-312 (2020)

4. Ammar, F., Ejbehi, Z., Makhlouf, A.: Cohomology and deformations of Hom-algebras. J. Lie Theory 21(4), 813-836 (2011)

5. Ammar, F., Makhlouf, A.: Hom-Lie and Hom-Lie admissible superalgebras. J. Algebra 324, 1513-1528 (2010)

6. Ammar, F., Makhlouf, A., Saadaoui, N.: Cohomology of Hom-Lie superalgebras and q-deformed Witt superalgebra. Czech. Math. J. 63(3), 721-761 (2013)

7. Arnlind, J., Makhlouf, A., Silvestrov, S.: Ternary Hom-Nambu-Lie algebras induced by Hom-Lie algebras. J. Math. Phys. 51, 043515 (2010)

8. Arnlind, J., Makhlouf, A., Silvestrov, S.: Construction of $n$-Lie algebras and $n$-ary Hom-Nambu-Lie algebras. J. Math. Phys. 52, 123502 (2011)

9. Ataguema, H., Makhlouf, A., Silvestrov, S.: Generalization of n-ary Nambu algebras and beyond. J. Math. Phys. 50, 083501 (2009)

10. Bakayoko, I.: Laplacian of Hom-Lie quasi-bialgebras. Int. J. Algebra 8(15), 713-727 (2014)

11. Bakayoko, I.: L-modules, L-comodules and Hom-Lie quasi-bialgebras, African Diaspora. J. Math. 17, 49-64 (2014)

12. Bakayoko, I.: Modules over color Hom-Poisson algebras. J. Gen. Lie Theory Appl. 8, 1 (2014)

13. Bakayoko, I.: Hom-post-Lie modules, $\mathcal{O}$-operator and some functors. arXiv:1610.02845 [math.RA]

14. Bakayoko, I., Diallo, O.W.: Some generalized Hom-algebra structures. J. Gen. Lie Theory Appl. 9, 226 (2015)

15. Bakayoko, I., Bangoura, M.: Left-Hom-symmetric and Hom-Poisson algebras. Konuralp J. Math. 3(2), 42-53 (2015)

16. Bakayoko, I., Touré, B.M.: Constructing Hom-Poisson color algebras. Int. J. Algebra 13(1), 1-16 (2019)

17. Issa, A.N.: Hom-Akivis algebras. Comment. Math. Univ. Carol. 52(4), 485-500 (2011)

18. Benayadi, S., Makhlouf, A.: Hom-Lie algebras with symmetric invariant nondegenerate bilinear forms. J. Geom. Phys. 76, 38-60 (2014)

19. Chaichian, M., Ellinas, D., Popowicz, Z.: Quantum conformal algebra with central extension. Phys. Lett. B 248, 95-99 (1990)

20. Chaichian, M., Isaev, A.P., Lukierski, J., Popowic, Z., Prešnajder, P.: q-deformations of Virasoro algebra and conformal dimensions. Phys. Lett. B 262(1), 32-38 (1991)

21. Chaichian, M., Kulish, P., Lukierski, J.: $q$-deformed Jacobi identity, $q$-oscillators and $q$-deformed infinitedimensional algebras. Phys. Lett. B 237, 401-406 (1990)

22. Chaichian, M., Popowicz, Z., Prešnajder, P.: $q$-Virasoro algebra and its relation to the $q$-deformed KdV system. Phys. Lett. B 249, 63-65 (1990)

23. Curtright, T.L., Zachos, C.K.: Deforming maps for quantum algebras. Phys. Lett. B 243, 237-244 (1990)

24. Damaskinsky, E.V., Kulish, P.P.: Deformed oscillators and their applications. Zap. Nauch. Semin. LOMI 189, 37-74 (1991) (in Russian) [Engl. transl.: J. Sov. Math. 62, 2963-2986 (1992)]

25. Daskaloyannis, C.: Generalized deformed Virasoro algebras. Mod. Phys. Lett. A 7(9), 809-816 (1992)

26. Hounkonnou, M.N., Dassoundo, M.L.: Center-symmetric algebras and bialgebras: relevant properties and consequences. In: Kielanowski, P., Ali, S., Bieliavsky, P., Odzijewicz, A., Schlichenmaier, M., Voronov, T. (eds.) Geometric Methods in Physics, Trends in Mathematics, pp. 281-293. Birkhäuser, Cham (2016)

27. Hounkonnou M.N., Dassoundo M.L.: Hom-center-symmetric algebras and bialgebras. arXiv:1801.06539 [math.RA]

28. Gaparayi, D., Issa, A.N.: A twisted generalization of Lie-Yamaguti algebras. Int. J. Algebra 6(7), 339-352 (2012)

29. Elhamdadi, M., Makhlouf, A.: Deformations of Hom-alternative and Hom-Malcev algebras. Algebra Groups Geom. 28(2), 117-145 (2011)

30. Hartwig, J.T., Larsson, D., Silvestrov, S.D.: Deformations of Lie algebras using $\sigma$-derivations. J. Algebra 295, 314-361 (2006) [Preprint in Mathematical Sciences 2003:32, LUTFMA-5036-2003, Centre for Mathematical Sciences, Department of Mathematics, Lund Institute of Technology (2003)] 
31. Hu, N.: $q$-Witt algebras, $q$-Lie algebras, $q$-holomorph structure and representations. Algebra Colloq. 6(1), 51-70 (1999)

32. Kassel, C.: Cyclic homology of differential operators, the Virasoro algebra and a $q$-analogue. Commun. Math. Phys. 146(2), 343-356 (1992)

33. Kitouni, A., Makhlouf, A., Silvestrov, S.: On $(n+1)$-Hom-Lie algebras induced by $n$-Hom-Lie algebras. Georg. Math. J. 23(1), 75-95 (2016)

34. Larsson, D., Sigurdsson, G., Silvestrov, S.D.: Quasi-Lie deformations on the algebra $\mathbb{F}[t] /\left(t^{N}\right)$. J. Gen. Lie Theory Appl. 2, 201-205 (2008)

35. Larsson, D., Silvestrov, S.D.: Quasi-Hom-Lie algebras, central extensions and 2-cocycle-like identities. J. Algebra 288, 321-344 (2005) [Preprints in Mathematical Sciences 2004:3, LUTFMA-5038-2004, Centre for Mathematical Sciences, Department of Mathematics, Lund Institute of Technology, Lund University (2004)]

36. Larsson, D., Silvestrov, S.D.: Quasi-Lie algebras. In: Noncommutative Geometry and Representation Theory in Mathematical Physics. Contemp. Math., vol. 391, Amer. Math. Soc., Providence, pp. 241-248 (2005) [Preprints in Mathematical Sciences 2004:30, LUTFMA-5049-2004, Centre for Mathematical Sciences, Department of Mathematics, Lund Institute of Technology, Lund University (2004)]

37. Larsson, D., Silvestrov, S.D.: Graded quasi-Lie algebras. Czech. J. Phys. 55, 1473-1478 (2005)

38. Larsson, D., Silvestrov, S.D.: Quasi-deformations of $s l_{2}(\mathbb{F})$ using twisted derivations. Commun. Algebra 35, 4303-4318 (2007)

39. Liu, K.Q.: Quantum central extensions. C. R. Math. Rep. Acad. Sci. Can. 13(4), 135-140 (1991)

40. Liu, K.Q.: Characterizations of the quantum Witt algebra. Lett. Math. Phys. 24(4), 257-265 (1992)

41. Liu, K.Q.: The Quantum Witt Algebra and Quantization of Some Modules over Witt Algebra, PhD Thesis. Department of Mathematics, University of Alberta, Edmonton (1992)

42. Loday, J.-L.: Dialgebras. In: Lecture Notes in Mathematics, vol. 1763. Springer, Berlin, pp. 7-66 (2001) [Prépublication de 1'Inst de Recherche Math. Avancée (Strasbourg), 14 (1999)]

43. Loday, J.-L., Ronco, M.: Trialgebras and families of polytopes. In: Homotopy Theory: Relations with Algebraic Geometry, Group Cohomology, and Algebraic K-Theory, Contemp. Math., vol. 346. Amer. Math. Soc., Providence, pp. 369-673 (2004)

44. Ma, T., Zheng, H.: Some results on Rota-Baxter monoidal Hom-algebras. Results Math. 72(1-2), 145-170 (2017)

45. Ma, T., Makhlouf, A., Silvestrov, S.: Rota-Baxter cosystems and coquasitriangular mixed bialgebras. J. Algebra Appl. (2019) (accepted)

46. Ma, T., Makhlouf, A., Silvestrov, S.: Rota-Baxter bisystems and covariant bialgebras. arXiv:1710.05161 [math.RA]

47. Ma, T., Makhlouf, A., Silvestrov, S.: Curved $\mathcal{O}$-operator systems. arXiv:1710.05232 [math.RA]

48. Makhlouf, A.: Hom-dendriform algebras and Rota-Baxter Hom-algebras, in proceedings of international conferences in Nankai series in pure. In: Bai, C., Guo, L., Loday, J.-L. (eds.) Applied Mathematics and Theoretical Physics, vol. 2. World Scientific, Singapore, pp. 147-171 (2012)

49. Makhlouf, A., Silvestrov, S.D.: Hom-algebra structures. J. Gen. Lie Theory Appl. 2(2), 51-64 (2008) [Preprints in Mathematical Sciences 2006:10, LUTFMA-5074-2006, Centre for Mathematical Sciences, Department of Mathematics, Lund Institute of Technology, Lund University (2006)]

50. Makhlouf, A., Silvestrov, S.: Hom-Lie admissible Hom-coalgebras and Hom-Hopf algebras, chapter 17. In: Silvestrov, S., Paal, E., Abramov, V., Stolin, A. (eds.) Generalized Lie theory in Mathematics. Physics and Beyond, pp. 189-206. Springer, Berlin (2009)

51. Makhlouf, A., Yau, D.: Rota-Baxter Hom-Lie admissible algebras. Commun. Algebra 23(3), 1231-1257 (2014)

52. Munthe, H.K., Lundervold, A.: On post-Lie algebras. Lie Butcher series and moving frames. Found. Comput. Math. 13, 583-613 (2013)

53. Richard, L., Silvestrov, S.D.: Quasi-Lie structure of $\sigma$-derivations of $\mathbb{C}\left[t^{ \pm 1}\right]$. J. Algebra 319(3), 12851304 (2008)

54. Sheng, Y.: Representation of Hom-Lie algebras. Algebr. Reprensent. Theory 15(6), 1081-1098 (2012)

55. Sigurdsson, G., Silvestrov, S.: Lie color and Hom-Lie algebras of Witt type and their central extensions. In: Generalized Lie Theory in Mathematics, Physics and Beyond, pp. 247-255. Springer, Berlin (2009)

56. Sigurdsson, G., Silvestrov, S.: Graded quasi-Lie algebras of Witt type. Czech. J. Phys. 56, 1287-1291 (2006)

57. Vallette, B.: Homology of generalized partition posets. J. Pure Appl. Algebra 208, 699-725 (2007)

58. Yau, D.: Enveloping algebras of Hom-Lie algebras. J. Gen. Lie Theory Appl. 2(2), 95-108 (2008)

59. Yau, D.: Hom-algebras and homology. J. Lie Theory 19(2), 409-421 (2009)

60. Yau, D.: A Hom-associative analogue of Hom-Nambu algebras. arXiv:1610.02845v1

61. Yau, D.: Non-commutative Hom-Poisson algebras. arXiv:1010.3408 [math.RA] 
62. Yau, D.: Hom-bialgebras and comodule Hom-algebras. Int. Electron. J. Algebra 8, 45-64 (2010)

63. Yau, D.: On $n$-ary Hom-Nambu and Hom-Nambu-Lie algebras. J. Geom. Phys. 62, 506-522 (2012)

64. Yuan, L.: Hom-Lie color algebras. Commun. Algebra 40(2), 575-592 (2012)

Publisher's Note Springer Nature remains neutral with regard to jurisdictional claims in published maps and institutional affiliations. 